\title{
Anatomical Basis of Approaches to Liver Resection
}

\author{
Madhusudhanan Jegadeesan $^{1 *}$ and Ramprasad Jegadeesan ${ }^{2}$ \\ ${ }^{1}$ Hepatopancreaticobiliary and Liver Transplant Surgery Unit, Apollo Speciality \\ Hospitals, Madurai, Tamilnadu, India \\ ${ }^{2}$ Department of Gastroenterology, Southern Illinois University, IL, USA \\ *Corresponding Author: Madhusudhanan Jegadeesan, Consultant, \\ Hepatopancreaticobiliary and Liver Transplant Surgery Unit, Apollo Speciality \\ Hospitals, Madurai, Tamil Nadu, India.
}

Received: September 04, 2020

Published: November 25, 2020

(C) All rights are reserved by Madhusudhanan Jegadeesan and Ramprasad Jegadeesan.

\begin{abstract}
Essential to successful and safe performance of any liver resection is the understanding of anatomical aspects pertaining to liver resection. The different approaches to liver resections reflect the different techniques and surgical maneuvers executed at specific anatomical regions in and around liver. The three approaches to liver resection, as put forth by Claude Couinaud, are the intrafascial, extrafascial and the extrafascial transfissural approach. The later two approaches are essentially dissection of Glissonean pedicles at the hilum of the liver. This Glissonean pedicle approach is facilitated by newer insights into anatomy of liver gained by modern pathological and surgical research. The Glissonean pedicle approach is safe as it achieves inflow control early in the process of liver resection and ontologically sound as it is an anatomical approach. It is also effective when employed during minimally invasive liver resections. Both intrafascial and extrafascial approaches are relevant in specific circumstances of liver resection. This chapter will specifically analyse the anatomical basis that surrounds the approaches to liver resection.
\end{abstract}

Keywords: Anatomical Hepatectomy; Glissonean Pedicle; Leannec Capsule; Major Hepatectomy; Limited Resection

\section{Introduction}

With advancement in anaesthesia and critical care, hepatic surgery is now safely performed all over the world. Essential to the safe performance of hepatic surgery is the understanding of anatomical principles governing the operative procedure. From the time of Claude Couinaud who carefully dissected several liver specimens and proposed the segmental anatomy of the liver to the present date, our understanding of liver anatomy is getting refined. This chapter focuses on recent advancements in the knowledge of liver anatomy and its relevance in various approaches to liver resection.

\section{Anatomy of the glissonean pedicle}

The portal vein, hepatic artery and bile duct is enveloped in a dense connective tissue known as the glisson sheath. It wraps these structures and forms the glissonean pedicle which travels within the liver parenchyma along these structures. At the level of the liver hilum, this sheath condenses into a thick structure and forms what is called the hilar plate system. This sheath/plate system is vital in understanding approaches to liver resection [1].

\section{Approaches to the glissonean pedicle}

Anatomical liver resection is primarily an approach to the Glissonean pedicle. Ligation and division of glissonean pedicle is safe as it provides complete inflow control. Further, this approach is demonstrated to be superior from an oncological standpoint compared to a non-anatomical liver resection for hepatocellular carcinoma [2]. Glissonean pedicle approach gains further significance in the era of minimally invasive approaches like laparoscopic and robotic hepatectomies.

According to Coiunaud, there are three approaches to Glissonean pedicles [3]: the intrafascial, the extrafascial and the extrafascial and transfissural approach (Figure 1). 


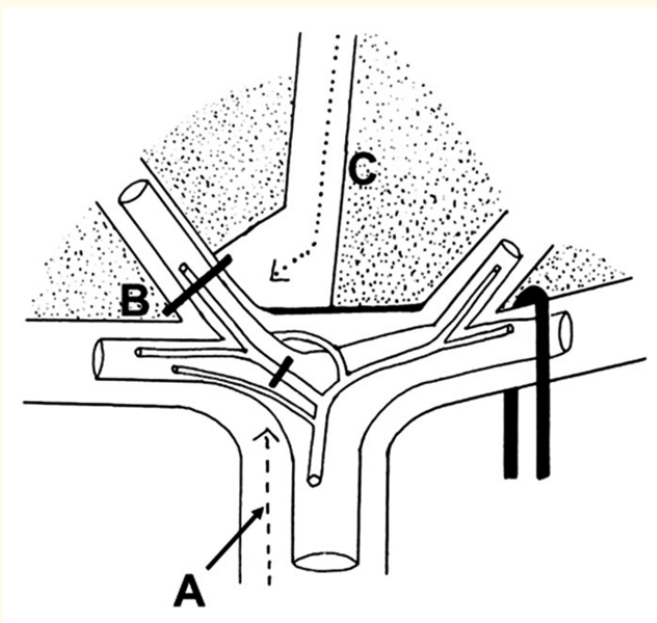

Figure 1: The three methods of access to the portal pedicle. A: Intrafascial approach. B: Extrafascial approach. C: Extrafascial and transfissural approach.

The intrafascial approach is isolation of individual vascular elements within the vasculobiliary sheath. Portal vein branches and hepatic artery to the hemiliver can be isolated by dissection within the hepatoduodenal ligament (Figure 2 and 3). It is also called the 'control method' and is introduced by Honjo and Lortat-Jacob in the 1950s [4,5]. In living donor liver transplantation, during donor hepatectomy, this approach will provide necessary length of these critical vascular structures for anastomosis during liver transplantation. However, this approach has a potential risk of injury to bile duct and vessels to the remaining liver due to failure to identify anatomical variations [6].

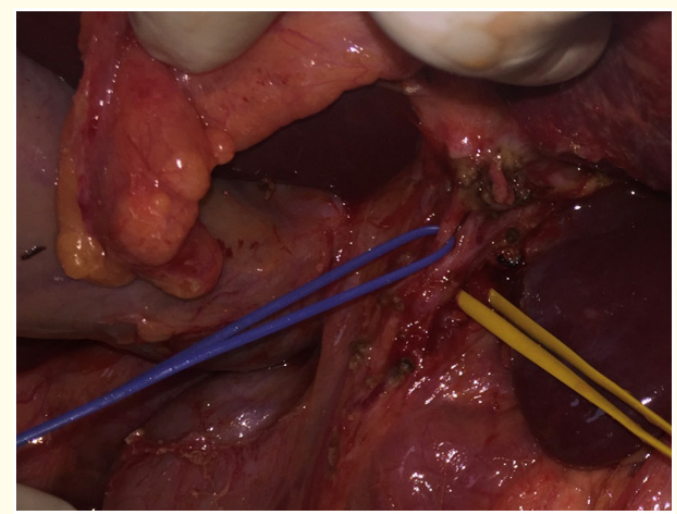

Figure 2: Intrafascial approach to left hepatectomy for hepatolithiasis. Left hepatic artery is looped with blue loop and left portal vein with yellow loop.

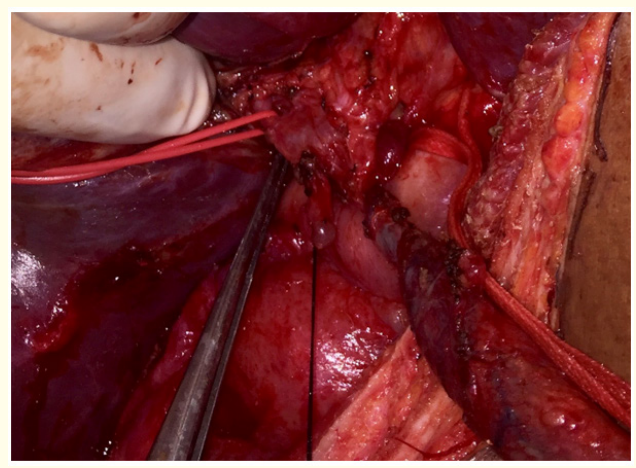

Figure 3: Isolation of right anterior sectoral artery by intrafascial approach. Note the gallbladder is pulled down for facilitating the dissection over the right anterior pedicle. The selective ligation of anterior sectoral artery was performed for a traumatic pseudoaneurysm of the right anterior hepatic artery occurring within the liver parenchyma.

The extrafascial approach is the actual glissonean pedicle approach. This approach entails isolation of the glissonean pedicle containing the portal vein, hepatic artery and the bile duct by going around the vasculobiliary sheath of Glisson. This can be done totally extrahepatically without destruction of liver parenchyma. It can also be done intrahepatically by a minor hepatotomy at the site of entry of these pedicles into the respective sectors or by a major liver transection through the main portal fissure (Cantlie's line) which then constitutes the 'extrafascial transfissural' approach.

\section{Intrahepatic approach with minor hepatotomy}

Minor hepatotomy can be done using digitoclasia as described by Galperin and Karagiulian [7] or using a clamp or vascular stapler as proposed by Machado and colleagues [8,9]. An intraoperative ultrasound can be used to guide the site of hepatotomy. Launois and Jamieson described hepatotomy above and below the hilum thereby gaining access to the pedicles by a blunt finger dissection through the hepatotomy site [10]. This technique has potential risk of unexpected injury to portal pedicle or hepatic veins.

Intrahepatic approach with major hepatotomy/extrafascial and transfissural approach

This approach was proposed by Couinaud in 1957 [11]. Later, Lin from Taiwan and Ton That Tung from Vietnam successfully performed liver resection using this approach (Figure 4) [12,13]. This is done by splitting the liver across the major portal fissure and once the dissection reaches the hilar plate, the surgeon can observe 
the origin Glissonean pedicles which can be looped and divided. A small variation of this technique was described in Japanese by Okamoto., et al. This technique entails transection of parenchyma of the segment 4 of liver above the hilar plate and gaining access to the glissonean pedicles. This was termed the 'unroofing method' [14].

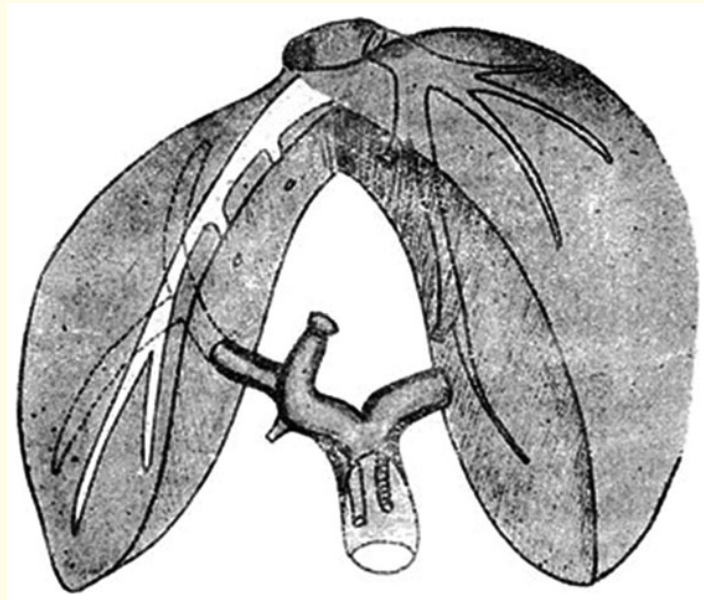

Figure 4: Anterior sectionectomy with the extrafascial and transfissural approach by Ton That Tung. (Adopted from Ton-That-Tung and Nguyen-Duong-Quang.; Presse Med. 1965;73:3015-7).

Extrahepatic glissonean pedicle isolation/extrafascial approach

In 1985, Couinaud demonstrated that left hepatectomy can be successfully performed by extra hepatic isolation of left Glissonean pedicle [15]. However, it is not known whether Couinaud actually performed an operation with this technique. Professor Takasaki from Japan popularised this technique (Figure 5) [16].

\section{Newer understanding of glissonean sheath system}

The sheath covering the vasculo-biliary structures to the liver was first described by Johannis Waleus in 1640 [17]. It was also described as a pedicle containing the portal vein, hepatic artery and the bile duct enveloped in a sheath by Glisson in 1642 [18]. Hence, it can be stated that the Glissonean Pedicle is wrapped by the Waleus sheath [19]. Centuries later, Laennec described a membrane covering the liver which became known as the Laennec capsule [20]. Furthermore, Couinaud introduced the concept of Hilar Plate system which he proposed to be the fibrous thickening of the sheath covering the Glissonean pedicle [21].

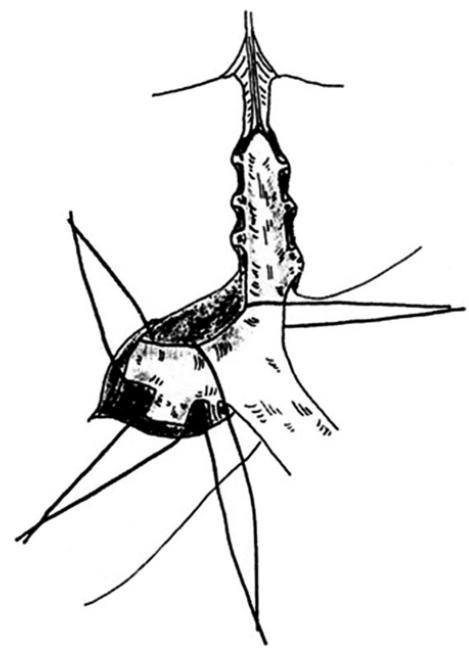

Figure 5: The extrafascial approach by Takasaki (Adopted from Takasaki K et al., Int Surg. 1990;75:73-7).

Couinaud also demonstrated that Laennec capsule is quite distinct from and not continuous with the glissonean sheath [3]. However, beyond this, the relationship between Leannec capsule and the plate/sheath system has never been highlighted in the literature. Nevertheless, understanding of this particular relationship will help in safe extrahepatic isolation of Glissonean pedicles.

An elegant histological study conducted by Hayashi., et al. on cadaveric livers using special elastic fiber and lymphatic vein staining revealed that Laennec capsule is a distinct fibrous layer covering the Glissonean pedicle (so called Glisson capsule) and extending up to the periphery of the pedicles [22]. Further study by Sugioka., et al. demonstrated that Laennec capsule is a dense fibrous layer beneath the serosa, extending on the surface of the bare area of liver and onto the cystic fossa beneath the cystic plate. Also, as stated before, Laennec capsule covers the liver above the hilar plate [23]. Hence, the concept of Laennec capsule helps in the newer understanding of the plate/sheath system (Figure 6). According to Sugioka and colleagues, at the level of liver hilum, there exists a potential space bound below by the Glissonean plate/sheath system and above by the Laennec capsule of the liver parenchyma which can be surgically developed to isolate the Glissonean pedicles. Understanding of this concept is critical to the performance of major and segmental liver resections by extrafascial extra hepatic Glissonean pedicle approach $[14,23]$. While performing an anatomical hepatectomy, these pedicles can be encircled without liver parenchymal dissection, ligated and divided as one bundle [24]. 


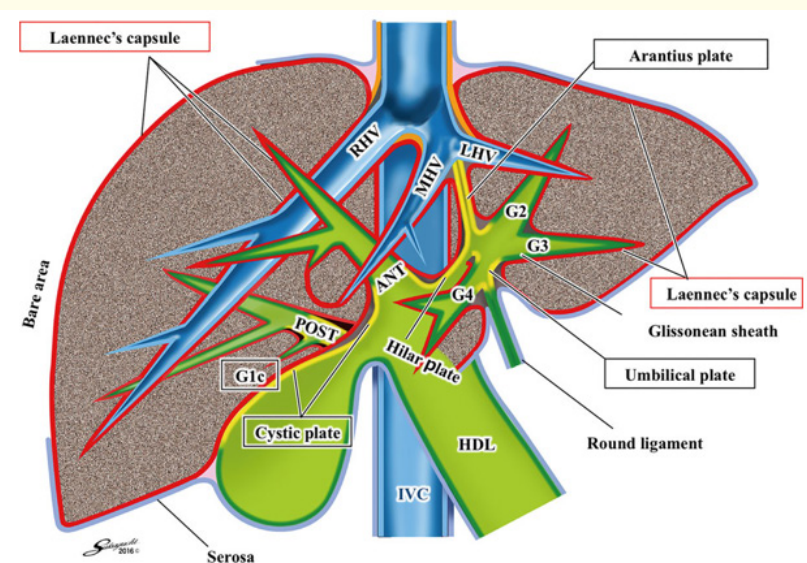

Figure 6: The schema of a novel comprehensive surgical anatomy of the liver based on Laennec's capsule. Laennec's capsule (red and orange line) covers not only the entire surface of the liver parenchyma beneath the serosa (blue line) including the bare area but also the intrahepatic parenchyma surrounding the Glissonean pedicles (green line) and the plate system (yellow line). Between the parenchyma of the cystic fossa and the subserosal layer of the gallbladder, Laennec's capsule and the cystic plate are interposed. There exists a gap (gray space) between Laennec's capsule and Glissonean pedicles (the plate system) that allows us to isolate the extrahepatic Glissonean pedicles. The red line between the peripheral Glissonean pedicle and the hepatic vein, and the orange line covering the suprahepatic inferior vena cava. (Adopted from Sugioka A et al., J Hepato-Biliary-Pancreat Sci. 2017;24:17-23).

\section{Six gates of Sugioka}

In an attempt to standardise the Glissonean pedicle approach to liver resection, Sugioka., et al. from Fujita University, Japan proposed six gates based on four anatomical landmarks. The four anatomical landmarks are: the Arantius plate, the umbilical plate, cystic plate and the caudate process pedicle. The gates are entry/ exit points on either side of these anatomical landmarks. The glissonean pedicle approach entails development of the potential space between the sheath of these anatomical landmarks and the Laennec capsule thereby all the 'six gates' are free to access (Figure 7). The preliminary step is to detach the upper surface of the hilar plate from the base of the segment 4 . This can be done because there are no branches that arise from the anterior margin of the hilar plate at this place. There are few caudate branches arising from the posterior margin of the plate which can be carefully li- gated. After this separation, the hilar plate can be pulled down from the quadrate lobe giving access to both the right and left pedicles. Lending credence to the above description, Laennec's capsule can be observed covering the surface of liver parenchyma after the separation of the hilar place.
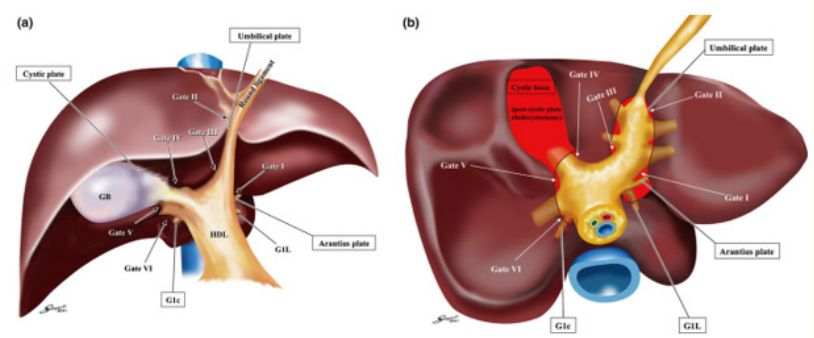

Figure 7: The schema of the four anatomical landmarks and six gates in the frontal view. For standardization of the extrahepatic Glissonean pedicle isolation, it is essential to recognize the four anatomical landmarks (enclosed texts) and six gates. (b) The schema of the six gates and Laennec's capsule in the caudal view. The schema shows the relationship between the six gates and Laennec's capsule (red area). The gaps between Laennec's capsule and the Glissonean pedicle (the plate system) could be entered only at these six gates. Gate I: the caudal end of the Arantius plate, Gate II: the junction between the round ligament and the umbilical plate, Gate III: the right edge of the Glissonean pedicle root of the umbilical portion (Gup: G2 + 3+4), Gate IV: the left edge of the posterior extremity of the cystic plate or the anterior Glissonean pedicle, Gate V: the bifurcation of the right main Glissonean pedicle, Gate VI: the space between the posterior Glissonean pedicle and the G1c (Adopted from Sugioka A et al., J HepatoBiliary-Pancreat Sci. 2017;24:17-23).

\section{Glissonean pedicle approach to left liver}

The first step in this approach is to detach the Arantius plate from the Laennec capsule at the base of the umbilical plate. The next step is to follow the round ligament and develop the space between the umbilical plate and the laennec capsule. This opens gates 1, 2 and 3. For an anatomical left lateral sectionectomy, a surgical loop can be passes across gates 1 and 2 and dividing the glissonean pedicles of segment 2 and 3. For an anatomical left hepatectomy, looping of the glissonean pedicle across the gates 1 and 3 will achieve total extrahepatic vascular isolation of the left liver. 


\section{Glissonean pedicle approach to right liver}

The first step in this approach is to perform a fundus-first cholecystectomy and detach the base of the gallbladder along with the cystic plate from the Laennec capsule of the cystic fossa. This exposes the right anterior pedicle which can be detached from the liver. The origin of the right posterior pedicle is generally visible behind the anterior pedicle at the Rouvier's sulcus. The glissonean pedicle to the caudate process is posterior and medial to the sulcus. Dissection of these structures from the adjacent Laennec capsule will provide access to the gates 4, 5 and 6. Extrafascial isolation of right anterior pedicle can be done by looping across gates 4 and 5 while posterior pedicle can be looped across gates 5 and 6 .

\section{Advantages of glissonean pedicle approach}

In case of small hepatocellular carcinomas, ligation of the portal pedicle of the segment bearing the small HCC, before the liver parenchymal dissection is a sound oncological practice. It has been shown by the Japanese groups that glissonean pedicle approach provides better outcomes compared to non-anatomical partial hepatectomy for small HCC [25,26]. Japanese surgical society guidelines also advocates anatomical hepatectomy for hepatocellular cancers [27].

This approach can be employed in laparoscopic or robotic liver resections as it helps in inflow control quite early during the surgery and maintains a bloodless field. It is especially useful in central hepatectomy and small anatomical liver resections in cirrhotic patients [28]. Furthermore, any anatomical variations in the vascular and biliary elements occur within the plate system. Hence, the glissonean pedicle approach has a significantly lesser risk of injury to these structures [14].

\section{Disadvantages of glissonean pedicle approach}

In a case of a hepatic tumor located at the hilum allowing the surgeon very little space for hilar dissection, or if the tumor invades the glissonean pedicle, this approach may not be possible. In these cases, the surgeon can resort to intrafascial approach or an extrafascial transfissural approach after a hepatic transection. In patients undergoing liver resection for hilar cholangiocarcinoma, this technique may not be feasible and needs careful hilar dissection for achieving oncological clearance.

\section{Approach to the tertiary pedicle}

Each second-order glissonean pedicle has 6 - 8 tertiary branches. The liver fed by a single tertiary branch is described as 'cone unit' by Professor Takasaki [28]. Each Couinaud segment has 3 - 4 cone units. This also corresponds to the recently described 1-2-20 configuration of liver wherein the main portal pedicle branches into right and left pedicles [29,30]. These second order pedicles have multiple branches resulting in up to 20 cone units.

Small liver resections and sub segmental resections can be anatomically performed by identifying the tertiary pedicles. In the left liver, this identification is fairly straightforward as it arises on either side of the umbilical fissure. On the right side of the liver, this can be done by first isolating the anterior and posterior sectoral pedicles. Vascular occlusion of a sectoral pedicle will help in demarcating the boundaries of the sector. The tertiary branches arising near the hepatic hilum can be ligated by further deep dissection along the sectoral pedicle. The tertiary branches arising from deep within the parenchyma can be isolated by parenchymal division along the ischemic line. The team led by Professor Makuuchi in Tokyo popularised the technique of intraoperative ultrasound-guided indigo carmine dye injection of subsegmental portal pedicles and resecting them [31,32]. Both Takasaki's technique of isolating the tertiary pedicle and Makuuchi's technique of dye injection enable performance of these small resections with adherence to oncological principles $[19,33]$. Further, a nationwide survey comparing anatomical resection and limited resection cases in Japan including more than 5500 cases, found significant difference in disease-free survival for tumors measuring 2 - $5 \mathrm{~cm}$ [34]. Hence, anatomical approaches to the tertiary pedicle bearing tumours measuring 2 - 5 $\mathrm{cm}$ results in optimal clinical outcomes.

\section{Conclusion}

There are several approaches to major and minor liver resections. Understanding of the anatomical basis of these approaches helps the surgeon choose a particular technique for a given patient. This also gains particular importance in the era of minimally invasive approaches to liver surgery.

\section{Bibliography}

1. Strasberg SM., et al. "Isolation of right main and right sectional portal pedicles for liver resection without hepatotomy or inflow occlusion". Journal of the American College of Surgeons 206 (2008): 390-396.

2. Yamazaki O., et al. "Comparison of the outcomes between anatomical resection and limited resection for single hepatocellular carcinomas no larger than $5 \mathrm{~cm}$ in diameter: a singlecenter study". Journal of Hepato-Biliary-Pancreatic Sciences 17 (2020): 349-358.

Citation: Madhusudhanan Jegadeesan and Ramprasad Jegadeesan. "Anatomical Basis of Approaches to Liver Resection". Acta Scientific Gastrointestinal Disorders 3.12 (2020): 17-23. 
3. Couinaud C. "The vasculo-biliary sheath". In: Surgical anatomy of the liver revisited. Paris (1989).

4. Lortat-Jacob JL and Robert HG. "Well defined technic for right hepatectomy". La Presse Médicale 60 (1952): 549-551.

5. Honjo I and Araki C. "Total resection of the right lobe of the liver; report of a successful case". The Journal of the International College of Surgeons 23.1-1 (1955): 23-28.

6. Bismuth H. "Surgical anatomy and anatomical surgery of the liver". World Journal of Surgery 6 (1982): 3-9.

7. Galperin EI and Karagiulian SR. "A new simplified method of selective exposure of hepatic pedicles for controlled hepatectomies". HPB Surgery: a World Journal of Hepatic, Pancreatic and Biliary Surgery's 1 (1989): 119-130.

8. Machado MAC., et al. "Intrahepatic Glissonian access for segmental liver resection in cirrhotic patients". The American Journal of Surgery 192 (2006): 388-392.

9. Machado MAC., et al. "Intrahepatic Glissonian approach for laparoscopic right segmental liver resections". The American Journal of Surgery 196 (2008): e38-e42.

10. Launois B and Jamieson GG. "The posterior intrahepatic approach for hepatectomy or removal of segments of the liver". Surgery, Gynecology and Obstetrics 174 (1992): 155-158.

11. Couinaud C., et al. "Le foie; études anatomiques et chirurgicales". Paris: Masson and Cie (1957).

12. Lin TY., et al. "Total right hepatic lobectomy for primary hepatoma”. Surgery 48 (1960): 1048-1060.

13. Tung TT and Quang ND. "Segmentary hepatectomy by transparenchymatous vascular ligation". La Presse Médicale 73 (1965): 3015-3017.

14. Okamoto E. "Hepatic resection for primary hepatocellular carcinoma: New trials for controlled anatomic subsegmentectomies by an initial suprahilar Glissonian pedicular ligation method (in Japanese)". Shokakigeka Seminar 23 (1986): 229241.

15. Couinaud CM. "A simplified method for controlled left hepatectomy". Surgery 97 (1985): 358-361.

16. Takasaki K., et al. "Highly anatomically systematized hepatic resection with Glissonean sheath code transection at the hepatic hilus". International Journal of Surgery 75 (1990): 73-77.
17. Walaeus J. "Epistolae duae de motu chyli et sanguinis ad Thomam Bartholeum". Leiden: Fransciscus Hackius (1640).

18. Glisson F. Anatomia hepatis. ap. Jo. Janss. a Waefberge (1665): 530.

19. Yamamoto M., et al. "Tips for anatomical hepatectomy for hepatocellular carcinoma by the Glissonean pedicle approach (with videos)". Journal of Hepato-Biliary-Pancreatic Sciences 21 (2014): E53-E56.

20. Laennec RTH. "Lettre sur des Tuniques qui enveloppent certains Visc eres, et fournissentdes gaines membraneuses a leurs vaisseaux". Journ De M ed Chir Et Pharm; Vend emiaire an XI (1802): 539-575.

21. Couinaud C. "Liver lobes and segments: notes on the anatomical architecture and surgery of the liver". La Presse Médicale 62 (1954): 709-712.

22. Hayashi S., et al. "Connective tissue configuration in the human liver hilar region with special reference to the liver capsule and vascular sheath". Japanese Society of Hepato-BiliaryPancreatic Surgery 15 (2008): 640-647.

23. Sugioka A., et al. "Systematic extrahepatic Glissonean pedicle isolation for anatomical liver resection based on Laennec's capsule: proposal of a novel comprehensive surgical anatomy of the liver". Journal of Hepato-Biliary-Pancreatic Sciences 24 (2017): 17-23.

24. Yamamoto M., et al. "Glissonean pedicle transection method for liver surgery (with video)". Journal of Hepato-Biliary-Pancreatic Sciences 19 (2012): 3-8.

25. Yamamoto M., et al. "Effectiveness of systematized hepatectomy with Glisson's pedicle transection at the hepatic hilus for small nodular hepatocellular carcinoma: retrospective analysis". Surgery 130 (2001): 443-448.

26. Yamamoto M., et al. "The value of anatomical liver sectionectomy for patients with a solitary hepatocellular carcinoma from 2 to $5 \mathrm{~cm}$ in greatest diameter". Journal of Surgical Oncology 100 (2009): 585-588.

27. Makuuchi M., et al. "Development of evidence-based clinical guidelines for the diagnosis and treatment of hepatocellular carcinoma in Japan". Hepatology Research 38 (2008): 37-51.

28. Takasaki K. "Glissonean pedicle transection method for hepatic resection: a new concept of liver segmentation". Japanese Society of Hepato-Biliary-Pancreatic Surgery 5 (1998): 286-291. 
29. Fasel JHD., et al. "Liver segments: an anatomical rationale for explaining inconsistencies with Couinaud's eight-segment concept". Surgical and Radiologic Anatomy 32 (2010): 761765.

30. Majno P., et al. "Anatomy of the liver: an outline with three levels of complexity--a further step towards tailored territorial liver resections". Journal of Hepatology 60 (2014): 654-662.

31. Makuuchi M., et al. "Ultrasonically guided subsegmentectomy". Surgery, Gynecology and Obstetrics 161 (1985): 346-350.

32. Makuuchi M., et al. "Surgery for small liver cancers". Seminars in Surgical Oncology 9 (1993): 298-304.

33. Hasegawa K., et al. "Prognostic impact of anatomic resection for hepatocellular carcinoma”. Annals of Surgery 242 (2005): 252-259.

34. Eguchi S., et al. "Comparison of the outcomes between an anatomical subsegmentectomy and a non-anatomical minor hepatectomy for single hepatocellular carcinomas based on a Japanese nationwide survey". Surgery 143 (2008): 469-475.

\section{Assets from publication with us}

- Prompt Acknowledgement after receiving the article

- Thorough Double blinded peer review

- Rapid Publication

- Issue of Publication Certificate

- High visibility of your Published work

Website: $\underline{w w w}$.actascientific.com/

Submit Article: www.actascientific.com/submission.php Email us: editor@actascientific.com

Contact us: +919182824667 